\title{
Propuesta de publicidad \\ y competitividad, caso: empresas Canaco Tampico
}

\author{
Javier Chávez Meléndez* \\ Nora Hilda González Durán" \\ Juan Carlos Guzmán García"*
}

\begin{abstract}
Resumen
LaCanacoesunaagrupacióndeempresas comerciales y de servicios que busca principalmente crear diferentes esquemas de apoyocomo micro, pequeñosy medianos empresarios. Un número importante de empresarios afiliados a la Canaco Tampico no tienen el conocimiento de algunas herramientas otécnicas de negocio, tales como la mercadotecnia y en su caso de la publicidad. Los negocios a nivel nacional son bastante competidos y difíciles por los escasos apoyos gubernamentales, además devariablescomolainseguridad que se vive en la zona sur de Tamaulipas. De forma teórica se basó en ocho modelos de competitividad y uno de medición de la publicidad. Para fines metodológicos se recurrió al diseño de una herramienta de acopio de información, basado en una revisión de la literatura. Entre los principales resultados obtenidos se encuentran los indicadores concernientes a la comunicabilidad, notoriedad y persuasión en las pautas publicitarias participantes.
\end{abstract}

\begin{abstract}
Canaco is agrouping of commercial and service companies that mainly seeks to create different support schemes such as micro, small and medium entrepreneurs. A significant number of employers affiliated to Tampico's Canaco have not the tools or knowledge of some business techniques, such as marketing and advertising your event. Businesses nationwide are pretty hard competitor for scarce government support, in addition to variables such as the insecurity prevailing in the southern part of Tamaulipas. Theoretically it was based on eight models of competitiveness and one measurement of advertising. For methodological purposes itwas used to design an information-gathering tool, based on a literature review. The main results obtained are the indicators concerning to the communicability, visibility and persuasion at participating advertising guidelines.
\end{abstract}

Palabras clave: competitividad, publicidad, mypes.

Keywords: competitiveness, publicity, smes.

Universidad Autónoma de Tamaulipas. Correo electrónico: jchavezm@uat.edu.mx

* Universidad Autónoma de Tamaulipas. Correo electrónico: nhgonzale@docentes.uat.edu.mx

*** Universidad Autónoma de Tamaulipas. Correo electrónico: jcguzman@uat.edu.mx 


\section{Introducción}

La publicidad es una herramienta de negocios muy antigua; la literatura señala que desde el instante en que los productos empezaron a comercializarse, surgió la necesidad de comunicar la presencia de los mismos

El desarrollo de las tecnologías de información y comunicación ha sido muy acelerado en los últimos años, lo que ha orillado a las empresas a la búsqueda de opciones cada día más complejas para difundir el conocimiento de sus productos De igual manera se han producido cambios en los hábitos de los consumidores, quienes han perdido interés en los medios de comunicación tradicionales

Los administradores de empresas mypes, porel nivel de especialización de sus negocios y por el tamaño de los mismos, en la mayoría de los casos no cuentan con conocimiento amplio de las técnicas y las formas de realizar la medición de la eficacia de su publicidad. Por consiguiente, las inversiones en publicidad en muchos de los casos ha derivados en esfuerzos infructuosos y costos elevados

Ante la situación antes expuesta, en donde cada día las empresas tienen que volverse más eficientes y escrupulosas en sus gastos, es que surge el interés por desarrollar esta investigación, buscando generar información que incida en la búsqueda constante de mejorar la competitividad de estos empresarios

\section{Desarrollo del tema}

\section{Variable de estudio: publicidad}

La American Marketing Association (ama) (1995) define a la publicidad como "cualquier forma pagada de presentación o promoción no personal, en favor de un patrocinador determinado". Kotler (2003: 470) la define de forma muy similar; Sánchez (1999:36) añade a la persuasión que influya hacia los consumidores; por otrolado, Treviño (2010:2) sostiene que la publicidad "es hablar decomunicación, de difundir un mensaje; es prometeralgoque más valecumplir; es buscarla preferencia del cliente" 
J Ferré (2005: 546) señala cuatro dimensiones del proceso publicitario:

La primera etapa del proceso se debe formalizar a través del briefing o brief, que es la preparación del documento completo con todo lo relacionado con la publicidad; la segunda es la planeación; la tercera corresponde a la ejecución, y la última se refiere al control y medición de resultados.

El éxito de los anuncios publicitarios, según Clow y Baack (2010: 120) consiste en tres ingredientes básicos: 1 Creación de un plan lógico de administración de la publicidad de la empresa 2 Diseño bien pensado de los anuncios 3 Selección cuidadosa de los medios De acuerdo con Erickson (1990: 47), la publicidad tiene dos funciones básicas: a) comunicar un mensaje, y b) crear una motivación de compra

De acuerdo con lo anterior los objetivos a medir de la publicidad son: el efecto en la comunicación y el efecto en las ventas. La eficacia publicitaria se refiere al nivel de alcance de los objetivos antes descritos.

Figura 1

Dimensiones de la variable eficacia publicitaria

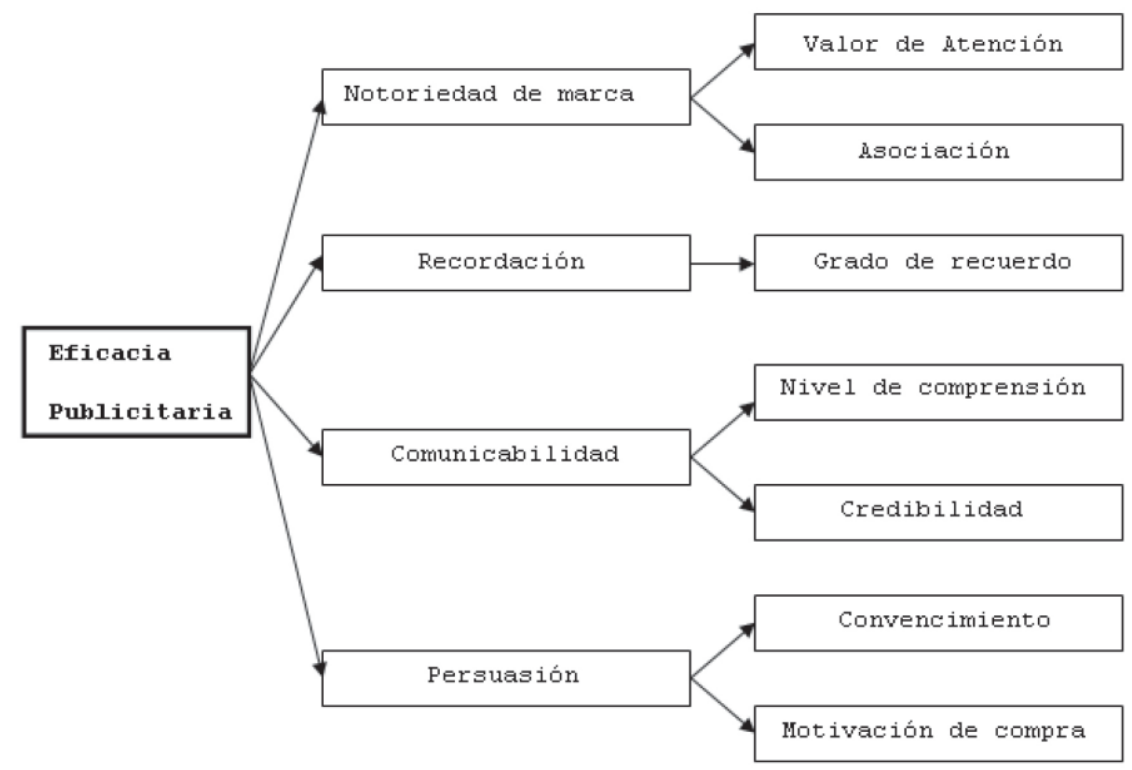

Fuente: autoría propia 


\section{Variable de estudio: competitividad}

Para medir la competitividad se recurrió a ocho diferentes modelos de la misma Sólo se consideraron modelos conceptuales dentro del nivel de análisis 1, que es el nivel de la empresa, de acuerdo con Abdel y Romo (2004), no empleando los demás niveles de análisis

\section{Modelos teóricos de competitividad empleados y sus dimensiones}

A continuación se describen los modelos teóricos de competitividad empleados y sus dimensiones conceptuales incluidas

B 1 Modelo nacional para la competitividad de mypes, del Instituto Nacional de Calidad, citado por Compite (2012) Este modelo incluye las siguientes dimensiones:

Conocimiento del entorno Alianzas Relación con clientes Recursos y actividades clave. Propuesta de valor Innovación Estructura de costos Fuente de ingresos

B2 Modelo de la competitividad sistémica (Esser, Wolfgang, Dirk, MeyerStamer, 1996) De este modelos se toman las dimensiones del nivel micro, que son:

Capacidad de la gestión Estrategias empresariales Gestión de la innovación Mejores prácticas en el ciclo de producción Integración en redes de cooperación tecnológicas Logística empresarial

B 3 Modelo de la competitividad integral, de López, López y Pérez (2004) De este planteamiento se consideraron las variables denominadas: papel del gobierno Acciones de la empresa.

B 4 Modelo de las matrices de prácticas de clase mundial, de Arechavala (1998) De este planteamiento teórico se tomaron:

Los objetivos, la toma de decisiones, el conocimiento del cliente, y el uso de la información financiera. 
B5 Modelo del análisis de la competitividad, de Ten Kate (citado por Susana Garduño, et al (2006) De este modelo se incluyen las dimensiones:

Factores que afectan los costos de los insumos, que inciden en sus niveles de precio, su rentabilidad, su permanencia y penetración en los mercados

Factores que determinan la eficiencia con que se utilizan los insumos.

Factores vinculados con precios, calidad y diferenciación de los productos

Factores que inciden en la diferenciación de productos o servicios

B 6 Modelo de competitividad en el comercio internacional, de Lerma (2000) De aquí fue posible emplear los elementos del nivel microestructural, que son:

Producto vendible en el mercado exterior (diseño, calidad, presentación, tecnología, envase, embalaje, precio) Comercialización (promoción, venta, servicio, condiciones comerciales). Empresa (principalmente en cuanto a su organización, capacidad económica, productiva y tecnológica, y actitud hacia los negocios internacionales)

B7 Modelo de desarrollo y generación de competitividad internacional, de Batres y García (2006) De esta formulación teórica se hacen participar los elementos:

Desarrollo de cadenas productivas. Capital humano, en cuanto a su capacitación y desarrollo.

B 8 Modelo de las ventajas competitivas, de Michael Porter (2010) De este modelo se manejaron las dimensiones:

Composición del sector. Poder e influencia de cada fuerza. Posición competitiva

\section{Metodología}

El presente proyecto se desarrolló con enfoque cuantitativo, con características de estudio descriptivo, transversal y no experimental

En consideración a las dimensiones de la publicidad descritas en el desarrollodel tema (notoriedad de marca, recordación, comunicabilidad y persuasión) se construyó un instrumento de medición basado en preguntas, toman- 
do en cuenta las siete subdimensiones mostradas en la figura 1, a partir de las cuales se estructuraron 20 ítems, manteniendo como referencia la escala de Likert con cuatro opciones

La validación del instrumento se llevó a cabo por medio de una prueba piloto, por la técnica de validación interna, por eliminación de sesgos en la aplicación del mismo y con la prueba alpha de Cronbach

Población y muestra Se tomó como población a las empresas mypes comerciales afiliadas a Canaco Tampico y la muestra se integró con 50 empresas. Por dificultades de acceso a las mismas la selección de las empresas siguió un proceso noaleatorio, finalmente ajuiciodel investigador.

Como unidades de análisis se consideró a cada empresa visitada y como sujeto de estudio a los administradores o gerentes de la empresa

Análisis de datos Se desarrolló empleando las herramientas de la estadística descriptiva y pruebas dehipótesis

De los modelos antes escritos se emplearon las dimensiones "escritos con letra cursiva", las cuales guardan una relación entre el dominio y conocimiento de la publicidad y su efecto en la competitividad

El estudio se basó en la siguiente lógica de investigación:

Se determinaron las dimensiones de la competitividad relacionadas con acciones de publicidad y comunicación comercial, tomando como premisa medirlaeficacia delapublicidad.

\section{Conclusiones}

\section{Notoriedad de marca}

El cuadro 1 se obtuvo como resultado de la investigación descriptiva de la muestra. En este cuadro se define cómo el público valora la dimensión de la eficacia publicitaria denominada "atención". Se observa que los valores en cuanto a la "atención" son favorables en la publicidad hecha por estas empresas 
Cuadro 1

Evaluación de la dimensión: "Atención"

\begin{tabular}{l|c|c|c|c}
\hline Atención & $\begin{array}{c}\text { Totalmente } \\
\text { de acuerdo }\end{array}$ & $\begin{array}{c}\text { De } \\
\text { acuerdo }\end{array}$ & $\begin{array}{c}\text { En } \\
\text { desacuerdo }\end{array}$ & $\begin{array}{c}\text { Totalmente } \\
\text { en desacuerdo }\end{array}$ \\
\hline $\begin{array}{l}\text { Elanuncio era muy divertido dever } \\
\text { y de escuchar. }\end{array}$ & $42 \%$ & $39 \%$ & $10 \%$ & $9 \%$ \\
\hline $\begin{array}{l}\text { Piensa que era bastante ingenioso y } \\
\text { divertido. }\end{array}$ & $14 \%$ & $76 \%$ & $7 \%$ & $3 \%$ \\
\hline $\begin{array}{l}\text { Las personas u objetos que } \\
\text { aparecieron en el anuncio llamaron } \\
\text { su atención. }\end{array}$ & $7 \%$ & $83 \%$ & $9 \%$ & $1 \%$ \\
\hline
\end{tabular}

Fuente: autoría propia

En el cuadro 2 se muestra cómo los clientes perciben la dimensión asociación de los mensajes publicitarios emitidos Se observa que hay subdimensiones dignas de atenderse, como el hecho de que el $89 \%$ considera que se hace lo mismo de siempre

Cuadro 2

Evaluación de la dimensión: "Asociación"

\begin{tabular}{l|c|c|c|c}
\hline Asociación & $\begin{array}{c}\text { Totalmente } \\
\text { de acuerdo }\end{array}$ & $\begin{array}{c}\text { De } \\
\text { acuerdo }\end{array}$ & $\begin{array}{c}\text { En } \\
\text { desacuerdo }\end{array}$ & $\begin{array}{c}\text { Totalmente en } \\
\text { desacuerdo }\end{array}$ \\
\hline $\begin{array}{l}\text { Relata lo que a veces siente o tiene } \\
\text { que ver con sus necesidades. }\end{array}$ & $52 \%$ & $37 \%$ & $6 \%$ & $5 \%$ \\
\hline $\begin{array}{l}\text { Es la clase de anuncio que siempre } \\
\text { se ha hecho, es lo mismo de } \\
\text { siempre. }\end{array}$ & $16 \%$ & $73 \%$ & $9 \%$ & $2 \%$ \\
\hline $\begin{array}{l}\text { Requiere mucho esfuerzo para } \\
\text { poder seguirlo. }\end{array}$ & $19 \%$ & $26 \%$ & $29 \%$ & $26 \%$ \\
\hline
\end{tabular}

Fuente: autoría propia

En el cuadro 3 se muestran los resultados de la dimensión "Recordación" En este cuadro se pueden señalar algunos datos, como el hecho de que el 90\% está cansado de ver estos anuncios Falta innovación, mejora ocambios 
Cuadro 3

Evaluación de la dimensión: "Recordación"

\begin{tabular}{l|c|c|c|c}
\hline Recordación & $\begin{array}{c}\text { Totalmente } \\
\text { de acuerdo }\end{array}$ & $\begin{array}{c}\text { De } \\
\text { acuerdo }\end{array}$ & $\begin{array}{c}\text { En } \\
\text { desacuerdo }\end{array}$ & $\begin{array}{c}\text { Totalmente en } \\
\text { desacuerdo }\end{array}$ \\
\hline Recordación & $34 \%$ & $56 \%$ & $8 \%$ & $2 \%$ \\
\hline $\begin{array}{l}\text { Lo había visto muchas veces, está } \\
\text { cansado de verlo. }\end{array}$ & $8 \%$ & $3 \%$ & $63 \%$ & $26 \%$ \\
\hline $\begin{array}{l}\text { Es un anuncio inusual, está } \\
\text { seguro de haber visto antes uno } \\
\text { como éste. }\end{array}$ & $12 \%$ & $23 \%$ & $36 \%$ & $29 \%$ \\
\hline
\end{tabular}

Fuente: autoría propia

Acontinuación se presenta el cuadro 4, en el cual se detallan las conclusiones más importantes del estudio

Cuadro 4

Principales conclusiones de la investigación

\begin{tabular}{l|l}
\hline Principales conclusiones & Explicación \\
\hline $\begin{array}{l}\text { Notoriedad. Las marcas no son lo } \\
\text { suficientemente reconocidas, por sus } \\
\text { anuncios. }\end{array}$ & $\begin{array}{l}\text { El posicionamiento de las marcas resulta } \\
\text { más por el consumo propio de los } \\
\text { productos de cada encuestado. }\end{array}$ \\
\hline $\begin{array}{l}\text { Recordación. En su mayoría muestran el } \\
\text { mismo formato y carecen del elemento } \\
\text { creativo para poder diferenciarse entre sí. }\end{array}$ & $\begin{array}{l}\text { No son lo suficientemente recordadas por } \\
\text { representan o innovación en presentarel } \\
\text { contenido. }\end{array}$ \\
\hline $\begin{array}{l}\text { Comunicabilidad. El mensaje resultó } \\
\text { apropiado, pero sin embargo no llega a } \\
\begin{array}{l}\text { adaptarse óptimamente al uso del medio } \\
\text { publicitario. }\end{array}\end{array}$ & $\begin{array}{l}\text { Se logra un buen entendimiento de la } \\
\text { información transmitida, pero en algunos } \\
\text { casos llega a sofocar por el exceso de } \\
\text { gráficos y déficit de tiempo. }\end{array}$ \\
\hline $\begin{array}{l}\text { Persuasión. En su mayoría el mensaje } \\
\text { induce y causa una reacción favorable, } \\
\text { pero no llega a ser determinante para la } \\
\text { compra. }\end{array}$ & $\begin{array}{l}\text { La decisión de compra es motivada por } \\
\text { presentar alguna promoción por gusto } \\
\text { del cliente o recomendación de boca a } \\
\text { boca. }\end{array}$ \\
\hline
\end{tabular}

Fuente: autoría propia 
Respuesta al objetivo general de la investigación

Determinar el grado de eficacia de la publicidad en empresas afiliadas a la Canaco Tampico

- Una vez realizadas las pruebas de hipótesis en función de los resultados obtenidos, se pueden resumir las conclusiones de estainvestigación, considerando que la eficacia de la publicidad en estas empresas está en un grado bajo-medio

- Una de las principales observaciones es la forma casera o "semiprofesional" como se producen las pautas publicitarias en las empresas estudiadas, así como la escasa planeación para su producción y distribución

- Adicionalmente se observó el desconocimiento de las formas de medición de la eficacia publicitaria.

\section{Recomendaciones}

Con base en los resultados obtenidos se ofrecen una serie de propuestas, las cuales se detallan a continuación:

a) Propuesta de desarrollo gerencial de los administradores de las empresas en estudio

Estructurar un programa de capacitación y asesoría para estos administradores, con la finalidad de prepararlos en las dimensiones de eficacia publicitaria y competitividad estudiadas

b) Asesoría de profesionales de la comunicación y la publicidad que emplean métricas de lo que estadísticamente impacta a la audiencia

c) En cuanto a los aportes que esta investigación puede beneficiar a la medición de la eficacia publicitaria en las empresas, es sin duda por medio del instrumentousado

d) Se ofrece el cuadro siguiente, que compila estudios de eficacia publicitaria como guía de apoyo a los administradores autodidactas Lo anterior como una guía de apoyo a los administradores 


\section{Cuadro 5}

Compilación de estudios de eficacia publicitaria

\begin{tabular}{l|l|l}
\hline Nombre del estudio & Objetivo & Principal característica \\
\hline $\begin{array}{l}\text { Dayafter recall test. Autor: } \\
\text { Burke, eua, 1995. }\end{array}$ & $\begin{array}{l}\text { Analizar la eficacia en pautas } \\
\text { publicitarias en medios } \\
\text { audiovisuales. }\end{array}$ & $\begin{array}{l}\text { Posprueba para medir } \\
\text { el grado de eficacia una } \\
\text { vez publicado el anuncio } \\
\text { audiovisual. Se realizan } \\
\text { entrevistas. }\end{array}$ \\
\hline $\begin{array}{l}\text { Eficacia de las campañas } \\
\text { publicitarias. Autor: } \\
\text { Alianza España, 2012. }\end{array}$ & $\begin{array}{l}\text { Análisis de las campañas } \\
\text { publicitarias sociales de } \\
\text { seguridad vial en España. }\end{array}$ & $\begin{array}{l}\text { Técnicas para evaluar la } \\
\text { conciencia social y difusión } \\
\text { de la campaña enjóvenes } \\
\text { españoles. }\end{array}$ \\
\hline $\begin{array}{l}\text { Estudio de efectividad } \\
\text { publicitaria online. Autor: } \\
\text { iab México, 2010. }\end{array}$ & $\begin{array}{l}\text { Conocer el impacto quetuvo } \\
\text { la campaña en términos del } \\
\text { conocimiento de la marca, } \\
\text { la intención de compra y su } \\
\text { posicionamiento dentro del } \\
\text { mercado. }\end{array}$ & $\begin{array}{l}\text { Métricas de conocimiento } \\
\text { de la marca, la asociación } \\
\text { que se crea con el mensaje, } \\
\text { lapreferencia por la marca } \\
\text { publicitada, y la intención } \\
\text { de compra. }\end{array}$ \\
\hline
\end{tabular}

Fuente: autoría propia

\section{Referencias bibliográficas}

Abdel, G, y Romo, D(2004) Documentos de trabajo en estudios de competitividad Centro de Estudios de Competitividad México: itam

American Marketing Association (1995) Dictionary of Marketing Terms, $2^{\text {a }}$ edición Estados Unidos: ntc Business Book

Arechavala, R (1998) "Formas de operación de las pequeñas empresas deloccidente de México: Los retos de la competitividad en mercados abiertos", Contaduría y Administración, núm 189

Batres, R , y García-Calderón, L (2006) Competitividad y desarrollo internacional. Cómo lograrlo en México México: McGraw-Hill

Clow, K, y Baack, D (2010) Publicidad, promoción y comunicación integral en marketing, $4^{\mathrm{a}}$ edición México:PearsonEducación

Compite (2012) Modelo nacional de competitividad para micro y pequeñas empresas Consultado en: http: Ilwww compite org mx/eventos/2012/cic_mendoza pdf

Erickson, B F (1990) Cómo dominar la publicidad. España: Norma

Esser, K., Wolfgang, H., Dirk, M., y Jörg, M. (1996). Systemic Competitiveness, New Governance Patterns for Industrial Development. Londres: die

Ferré, J (2005) Enciclopedia de marketing y ventas España: Océano/Centrum 
Garduño, S, Castro, M , y Rojas, J (2006) Redes organizacionales de la industria chocolatera delDistrito Federal yárea metropolitana de la ciudad de México y la competitividad de sus empresas Ponencia X Congreso Anual de la Academia de Ciencias Administrativas San Luis Potosí, México

Kotler, P, y Armstrong, G (2003) Fundamentos de marketing, 6 6 edición México: Prentice Hall

Lerma, A (2000) Comercio internacional. Metodología para la formulación de estudios de competitividad empresarial. México: ecafsa, pp 182-212

López, Martha, López, Maricela, y Pérez, Silvia (2004) Hacia una competitividad integral de las pymes en la era del conocimiento. Ponencia Congreso Acacia, mayo 2004

Porter, Michael (2010) Ventaja competitiva Creación y sostenibilidad de un rendimiento superior España: Pirámide

Sánchez, M (1999) Eficacia publicitaria: Teoría y práctica España: McGraw-Hill/Interamericana

Treviño, R (2010) Publicidad. Comunicación integral en marketing, 3ª edición. México: McGraw-Hill/Interamericana 